\title{
Benchmarking the grasping capabilities of the iCub hand with the YCB Object and Model Set
}

\author{
Lorenzo Jamone $^{1}$, Alexandre Bernardino ${ }^{1}$, and José Santos-Victor ${ }^{1}$
}

\begin{abstract}
The paper reports an evaluation of the iCub grasping capabilities, performed using the YCB Object and Model Set. The goal is to understand what kind of objects the iCub dexterous hand can grasp, and with what degree of robustness and flexibility, given the best possible control strategy. Therefore, the robot fingers are directly controlled by a human expert using a dataglove: in other words, the human brain is employed as the best possible controller. Through this technique, we provide a baseline for researchers who want to evaluate the performance of their grasping controller. By using a widespread robotic platform and a publicly available set of objects, we believe that many researchers can directly benefit from this resource; moreover, what we propose is a general methodology for benchmarking of grasping and manipulation that can be applied to any dexterous robotic hand.
\end{abstract}

Index Terms-Grasping, Dexterous Manipulation, Humanoid Robots, Multifingered Hands.

\section{INTRODUCTION}

B ENCHMARKING is fundamental for any activity of research and development, to evaluate intermediate results and to guide innovation. While this is a common practice in many fields, ranging from automated reasoning (e.g. for planning [1]) to computer vision (e.g. for object recognition [2, Chapter 12.1] or pedestrian detection [3]), it is still not widespread in robotics [4].

A common approach in computer vision benchmarks is to compare the machine performance to the human performance in a specific task, given the same visual input (i.e. the same images). For example, the images of a pedestrian detection dataset are labeled by a human to determine whether a pedestrian is present in each image and where; the performance of a computer vision algorithm is then compared to this baseline. In the robotics domain the definition of a human baseline is more challenging, because the tasks do not involve only perception, but also action: decision, planning, control. The mechanical properties of the hardware are critical: it would be

Manuscript received: August, 27, 2015; Revised November, 20, 2015; Accepted December, 17, 2015.

This paper was recommended for publication by Editor Han Ding upon evaluation of the Associate Editor and Reviewers' comments. This work was partially funded by the EU Projects LIMOMAN [PIEF-GA-2013-628315] and POETICON++ [FP7-ICT-288382].

${ }^{1}$ L. Jamone, A. Bernardino and J. Santos-Victor are with the Instituto de Sistemas e Robótica, Instituto Superior Técnico, Lisbon, Portugal ljamone, alex, jasv at isr.tecnico.ulisboa.pt

Digital Object Identifier: see top of this page.

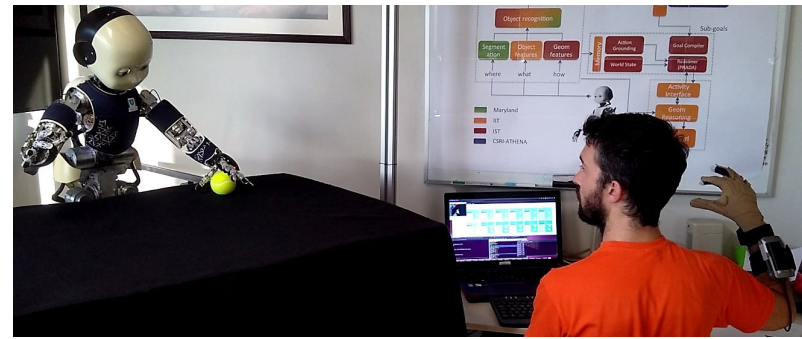

Fig. 1. Experimental setup. The fingers of the iCub robot hand (on the left) are controlled by a human operator with the CyberGlove-II dataglove (on the right).

unfair (and not very useful) to simply compare to the human performance in the same task, since the robot hardware and the human hardware are too different. Moreover, it is hard to propose benchmarks that can be performed by a large number of researchers, mainly because of the lack of common hardware and the scarce availability of public datasets. For example, while the digital images used for computer vision benchmarks can be easily made available to many researchers (e.g. on the web), it might be hard to retrieve exactly the same objects used in a robotic manipulation experiment.

Recently, a notable effort was made in this direction, with the creation of the YCB (Yale-CMU-Berkeley) Object and Model Set [5], [6]. Differently from previous attempts, this dataset does not only include 3D models of a large number of objects, but also the real physical objects are made available. This is very important for the benchmarking of robotic grasping and manipulation, because it allows to replicate the real world experiments, and not just the simulations.

In terms of common robotic platforms, one renowned example is the iCub humanoid robot [7], shown on the left side of Fig. 1 while grasping a tennis ball with its anthropomorphic hand. The robot is the outcome of the EU Project RobotCub (2005-2010), and it has been designed to be a shared robotic platform to study embodied cognition. Currently, more than 25 laboratories worldwide have their own iCub robot, and use it for research. Moreover, many ongoing EU Projects employ this humanoid robot as experimental platform (e.g. Poeticon++, TacMan, CoDyCo).

Although many research groups are currently working on the development of grasping and manipulation strategies using the iCub [8]-[15], still no benchmark is available 
to test the performances of the proposed controllers. The evaluation is therefore performed each time with different objects, procedures and performance metrics.

How to claim that a controller is good or very good? How to replicate the experiments to drive future development?

The objective of this paper is to define a baseline for grasping control by fixing both the hardware (the iCub hand) and the dataset (the YCB objects). A human user directly controls the fingers of the iCub using a dataglove, to grasp the YCB objects (as in Fig. 1). The user is familiar with the robot, with the dataglove and with the objects, and can see both the target object and the robot hand during the grasping actions. This provides a human baseline for the control, since the human brain is employed to solve the problems that an automatic controller would need to solve: e.g. how to use the visual feedback to drive the finger motion, how to coordinate the motion of the different fingers, how to select the best grasping strategy. We argue that this methodology could be applied to other widespread dexterous robot hands (e.g. Allegro, Shadow, Shunk SVH), using the same set of objects, both to compare the different robots and to define specific baselines for researchers who are testing their controllers with such robots.

Overall, we provide three main contributions. First, we restrict the YCB Object and Model Set to the set of objects that the iCub can physically grasp; this allows researchers to efficiently use the YCB objects for any manipulation task involving the iCub. Second, we perform an adaptation of the Gripper Assessment Benchmark proposed in [5] with the iCub hand on two sets of objects (a basic set and an extended set); this provides researchers who want to evaluate the performance of their grasp controllers on the $\mathrm{iCub}$ with a baseline to compare to. Third, we perform a more indepth evaluation on a single object, to test the robustness with respect to object displacements and rotations; we aim to show how the large number of DOFs of the iCub hand (19 joints actuated by 9 motors) offers a high grasping flexibility, that can be very useful even in simple grasping tasks, for example to compensate for reaching errors that may arise from imprecise robot calibration.

The rest of the paper is organized as follows. In Section II we describe the experimental setup, providing details about the robot, the dataglove and the objects set. The protocols followed in the evaluation experiments are discussed in Section III, and in Section IV we summarize the results. Finally, we provide some concluding remarks in Section V, and we sketch the future research directions.

\section{EXPERIMENTAL SETUP}

In this Section we outline the setup used in our experiments (see Fig. 1). We first introduce the $\mathrm{iCub}$ humanoid robot (Section II-A) and in particular its dexterous hand. Then, we present the dataglove that we use to control the iCub fingers
(Section II-B), and we describe the objects of the YCB Object and Model Set (Section II-C).

\section{A. The iCub humanoid robot}

The iCub is the main outcome of the EU Project RobotCub (2005-2010): a common open-source platform for researchers in cognitive robotics. All mechanical and electronics CAD files and the associated documentation are released under the GPL, while the software and middleware infrastructure (YARP [16]) is licensed either as GPL or LGPL. Also, a large number of YARP-based software modules are available as open source, due to the constant effort of a growing community of robotics researchers, practitioners and enthusiasts [17]. The robot mechanical and electronic design is described in detail in [18].

The hand of the iCub has been designed to enable dexterous manipulation, as this capability is crucial for cognitive development. It is sized $50 \mathrm{~mm}$ in length, $60 \mathrm{~mm}$ in width and $25 \mathrm{~mm}$ in thickness, making it one of the smallest and most dexterous of its kind. It has 19 joints, but it is driven by only 9 motors: this implies that group of joints are underactuated and their movement is obtained with mechanical couplings. The motion of the proximal phalanx and medial and distal phalanges are independent for the thumb, the index and the middle finger. The ring and small finger motions are coupled and driven by a single motor. Finally, two motors, placed directly inside the hand assembly, are used for thumb opposition and fingers adduction/abduction. The angles between the phalanxes (i.e. joint angles) are sensed by 17 small custom magnetic angular position sensors. The palm and the fingertips are covered by a pressure sensitive skin. More details about the design of the iCub hand, including sensing and actuation, can be found in [19].

\section{B. The Cyberglove-II dataglove}

Datagloves have been used since about 30 years to measure human hand movements for a number of applications [20], including the control of robot hands [10], [21]-[23]. In our experiments we use the Immersion CyberGlove-II dataglove [24], which is considered the state-of-the-art input device for recording human hand postures [20]. The feasibility of using datagloves for robot hand control has been demonstrated both for the CyberGlove [10], [21], [22] and for custom devices that use the same sensing technology [23].

Our version of the CyberGlove-II has a total of 15 sensors (versions with 18 or 22 sensors also exists), with two flexure sensors per finger (total of ten sensors), three abduction sensors placed between the fingers, one sensor for the thumb opposition and one palm-flexure sensor (we do not use this latter sensor). Most of the sensors measurements are directly mapped to the 9 robot hand motors: 3 for the thumb (flexure of the two phalanxes and thumb opposition) and 2 for both 
the index and the middle fingers (flexure of the two phalanxes), for a total of 7 motors. Then, the measurements from the ring and little fingers (4 measurements) are combined together and mapped to the single motor that actuates the robot ring and little fingers. Finally, the measurements from the fingers abduction (3 measurements) are combined together and mapped to the single motor that actuates the robot fingers abduction. A user-dependent calibration is performed to optimize the mapping coefficients, which is described in detail in [10]. In short, a number of finger movements toward pre-defined hand postures are performed (e.g. hand fully open, hand fully closed), and the collected data is used to estimate the coefficients through least squares regression.

Clearly, the robotic mimicking of the human hand postures cannot be perfect, not only because of mapping inaccuracies, but mainly because of the structural differences between the human hand and the robot hand. However, the exact reproduction of human hand postures is not the goal of our system; the goal, instead, is to grasp and manipulate objects effectively. Therefore, the required feature is to be able to control all the joints of the robot fingers simultaneously over their entire range of motion, in a natural way. During the grasping actions the human user can see both the robot hand and the target object, both from their own perspective and from the robot perspective (images from one of the robot cameras are displayed on a screen behind the robot), and therefore they can exploit such visual feedback to shape the robot fingers on the object as desired, and also to correct the posture after grasping if slippage is visually detected. Moreover, all the experiments are performed by the same human user, who is very experienced in the use of the setup (both concerning the glove and the robot hand).

\section{The YCB Object and Model Set}

The YCB Object and Model Set [6] is a collection of daily life objects with different shape, size, texture, weight and rigidity. The associated dataset includes high-resolution RGBD scans, physical properties and geometric models of the objects. In addition, some example of benchmarking protocols are provided [5]. A total of 69 objects (or objects assortments) are divided in four main categories: food items (e.g. cracker box, chips can, plastic banana), kitchen items (e.g. sponge, fork, skillet), tool items (e.g. scissors, hammer, screwdriver) and shape items (e.g. tennis ball, foam brick, rope, an assortment of cups). An additional category, task items, comprises 6 objects that can be used for specific tasks (e.g. a 9-peg-hole test, an airplane toy to assemble); we do not consider these objects in this paper. The set is freely distributed to research groups worldwide at international workshops (our group acquired one for free at a ICRA 2015 workshop), or delivered at a reasonable cost.

\section{BENCHMARKING TESTS}

We perform three experiments, that are described in detail hereinafter. The first one aims to determine what objects within the YCB Object and Model Set can be grasped by the iCub (Section III-A); the outcome is a subset of the original YCB Set tailored to the $\mathrm{iCub}$ hand. The second one is an adaptation to dexterous hands of the Gripper Assessment Benchmark proposed in [5] (Section III-B); it provides a baseline for researchers who want to evaluate the performance of their grasping controller with the iCub. The third one consists in grasping one object under a wide range of pose uncertainties (Section III-C); the goal of this test is to benchmark the flexibility offered by the many DOFs of the dexterous iCub hand.

In all the experiments, the object to be grasped is placed on a table in front of the robot (see Figure 1). The surface of the table is $15 \mathrm{~cm}$ lower with respect to the origin of the robot root reference frame, which is located on the axis of rotation of the torso pitch in the middle of the legs (as defined in http://wiki.icub.org/wiki/ICubForwardKinematics). The object pose with respect to the robot is chosen so as to permit a successful arm reaching movement that does not approach joint limits or singular configurations. Before each grasping action the arm is in a fixed configuration, with the hand palm facing down, at a distance of about $25 \mathrm{~cm}$ from the table; we refer to this arm configuration as rest pose. For each object a default grasp pose is selected (i.e. position and orientation of the hand palm with respect to the object). In each grasping action, the robot automatically moves the arm from the rest pose to the grasp pose; then, the human user controls the robot fingers using the dataglove, to perform the grasping. While the rest pose is the same in all experiments, the grasp pose depends on the object. Videos of the experiments can be found online (http://limomanproject.blogspot.pt/p/videos.html).

Clearly, there are many hand poses that allows to grasp the object, and the selection of the default hand pose for a specific object has a large influence on the grasp success, robustness and flexibility. For example, the type and amount of object pose uncertainties the hand can deal with, are strongly affected by the default hand pose. The choice of the best hand pose depends on many variables: not only the kinematic and dynamic properties of the robot hand, but also the object properties (e.g. shape, weight, texture) and the strategy that will be employed to control the robot fingers. Since in our experiments the strategy to control the fingers will be selected in real-time by the human user, which is very familiar with the properties of both the hand and the objects, we exploit the human knowledge also for the selection of the default hand pose. The user physically moves the robot arm during an offline phase, until the best hand pose for the object is manually identified (i.e. kinesthetic demonstration); the correspondent arm configuration is stored as the grasp 


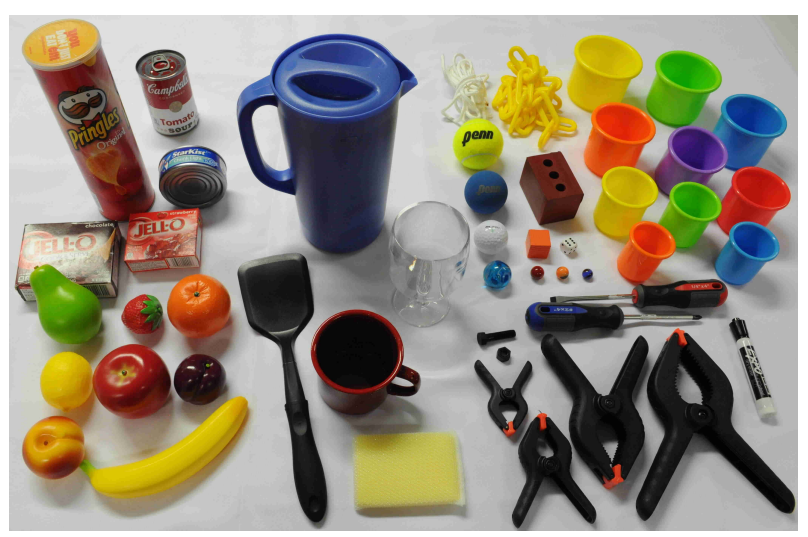

Fig. 2. Objects of the YCB Set that can be grasped with the iCub hand. On the left, food items. In the middle, kitchen items. On bottom right corner, tool items. On top right corner, shape items.

pose for that object, and it will be used for all the grasping actions directed to that object in that pose.

\section{A. Definition of the iCub YCB Object SubSet}

Each object of the original YCB Set [6] is placed on a table in front of the robot, in a pre-defined reachable pose for which a grasp pose was previously identified. For each object, the arm moves automatically from the rest pose to the grasp pose; then, the finger motion to grasp the object starts, controlled by the human user with the dataglove. Fingers control lasts 10 seconds at most. Then, the arm automatically moves back to the rest pose. If the object is successfully grasped, the user keeps controlling the robot fingers to hold the object in the hand while the arm is in the rest pose, for 5 seconds. Finally, the arm automatically moves back to the grasp pose, and the user opens the fingers to release the object. If the object is successfully held in the hand for at least 5 seconds, without any visible in-hand displacement, it is considered as graspable; otherwise, it is considered as non-graspable.

The image in Fig. 2 shows the objects of the YCB Set that are graspable with the iCub hand; the image in Fig. 3 shows the non-graspable ones. The representative grasp types employed are shown in Fig. 5; the grasps used with the other objects are small variations of these ones. Detailed information on each object (e.g. mass, size) can be found in [6].

\section{B. Gripper assessment}

This experiment is an adaptation to dexterous robot hands of the Gripper Assessment Benchmark (GAB) described in [5, Appendix B.2] .

The grasp procedure is the same of the previous experiment. However, each object is grasped in four different positions (SP1 = default position; $\mathrm{SP} 2=1 \mathrm{~cm}$ displacement on the $\mathrm{X}$ axis; $\mathrm{SP} 3=1 \mathrm{~cm}$ displacement on the $\mathrm{Y}$ axis; $\mathrm{SP} 4=1 \mathrm{~cm}$

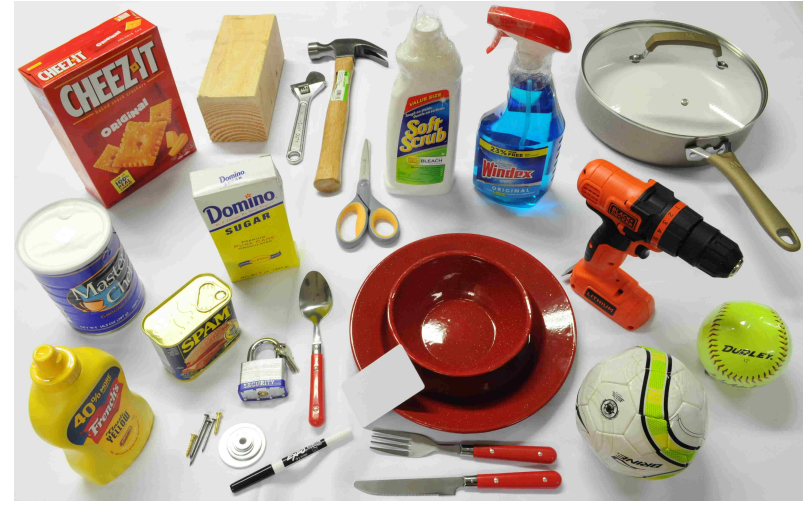

Fig. 3. Objects of the YCB Set that cannot be grasped with the iCub hand.

displacement on the $\mathrm{Z}$ axis). The grasp pose is the same for all SPs. For each grasp, 2 points are given if the objects is held in the hand for at least 3 seconds without visible motion, 1 point is given if some motion is detected, 0 points are given if the object drops. For articulated objects (rope and chain), the object is grasped and held for 20 times, each time from a random position around SP1; for each grasp, 0.5 points are given if no part of the object is touching the table while it is held ( 0 points otherwise).

The original GAB described in [5] is applied to robotic grippers; here we introduced a few differences to better fit the benchmark to dexterous hands.

In [5] the pose and motion of the gripper is the same in all SPs; instead, in our case the hand pose (i.e. the grasp pose) is the same, but the fingers motion can be different. This is meant to show that a dexterous hand with many joints/DOFs can cope with many object pose offsets, if supported by a smart controller. In our case the smart controller is provided by the human user, who is controlling the fingers with the dataglove, to provide the human baseline performance.

Moreover, differently from [5], in our test each grasp is performed with two different object orientations $\left(90^{\circ}\right.$ apart around the $\mathrm{Z}$ axis); this leads to a total of eight grasps for each object. This allows to benchmark the robustness not just to position offsets (as it is done in [5]), but also to orientation offsets.

In [5], after the object is grasped and lifted, it is also rotated by $90^{\circ}$ to prove grasp stability during motion. However, details about speed and acceleration of the object are not provided; even if they were provided, it would be difficult for researchers to replicate them exactly. Therefore, we removed this part in our experiments, and instead we test the grasp stability only by lifting the object from the table of about $25 \mathrm{~cm}$ and holding it against gravity for 3 seconds. The lifting movement is executed applying a bell-shaped velocity profile to the arm joints, that was resulting in a slow and smooth motion of the object, with a maximum speed in the middle of the motion of about $0.10 \mathrm{~m} / \mathrm{s}$. Any motion 


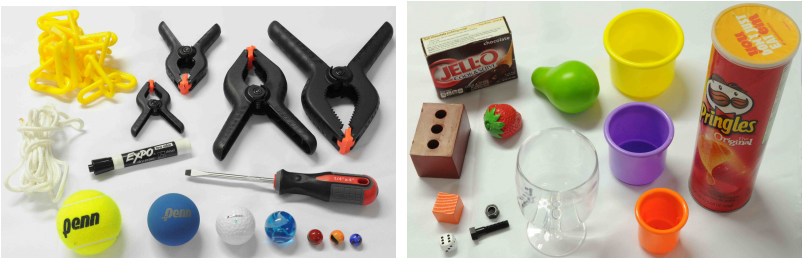

Fig. 4. Objects used for the Basic GAB (left image) and for the Extended $\mathrm{GAB}$ (right image). Left image, from left to right, top to bottom: chain, set of clamps ( $\mathrm{S}$ to $\mathrm{XL}$ ), rope, large marker, flat screwdriver, tennis ball, racquetball, golf ball, set of marbles (from XL to $\mathrm{S}$ ). Right image, from top to bottom, left to right: pudding box, foam brick, colored wood block, dice, plastic strawberry, plastic nut, plastic bolt, plastic pear, plastic wine glass, large cup $(100 \times 78 \mathrm{~mm})$, medium cup $(80 \times 70 \mathrm{~mm})$, small cup $(55 \times 60)$, chips can.

which is slow and smooth enough not to generate big and sudden accelerations would be appropriate to replicate the experiment: the only relevant source of perturbation of the object stability is the object weight. We perform two versions of the benchmark. The first one uses a subset of the objects used in [5], excluding the ones that are non-graspable for the iCub; we call this Basic GAB. The objects are shown in the left image in Fig. 4 and their names are reported in Table I. With respect to the GAB described in [5], we excluded all the flat objects (too thin), two round objects (soccer ball, too big, and softball, too heavy) and three tools (scissors, too thin and complex, hammer and driller, too heavy). The second version of the benchmark includes additional objects; we call this Extended $G A B$. We add three shape categories to the Basic set: cubic objects (pudding box, foam brick, colored wood block, dice), cylindrical objects (chips can, plastic wine glass, small cup, medium cup, large cup) and complex objects (plastic pear, plastic strawberry, plastic bolt, plastic nut). These objects are shown in the right image in Fig. 4 and their names are listed in Table II.

\section{Grasping flexibility}

This test is similar to the GAB, but is conducted with only one object, that is grasped 100 times after different pose offsets are applied with respect to the default pose (SP1); we call it Grasping Flexibility Benchmark (GFB). The object is first grasped at SP1, and then displaced on either $\mathrm{X}, \mathrm{Y}$ or Z, at each successive grasp attempt, with steps of $1 \mathrm{~cm}$. Maximum displacements are $\pm 5 \mathrm{~cm}$ on $\mathrm{X}$ and $\mathrm{Y}$, and \pm 2 $\mathrm{cm}$ on $\mathrm{Z}$. For each position (in total, 25 different positions), the object is grasped in 4 different orientations around the $\mathrm{Z}$ axis, separated $45^{\circ}$ apart; this makes a total of 100 grasps. All the other details of this test are the same as in the GAB, including the scoring.

\section{RESULTS AND DISCUSSION}

In this Section we start by providing a few general considerations on the grasping capabilities of the $\mathrm{iCub}$ hand
(Section IV-A), focusing in particular on the main reasons for grasp failures of the non-graspable object in Fig. 3. Then we show the results of the Gripper Assessment Benchmarks (Section IV-B) and of the Grasping Flexibility Benchmark (Section IV-C), and discuss them.

\section{A. General considerations on object graspability}

Based on our experiments, it is typically a combination of different object properties that determines the object graspability. However, weight and size appear to be the most important ones. Shape, texture and hardness also might play a role, when size and weight are close to the limits: compact shapes, rough textures and soft bodies are easier to grasp. The main categories of non-graspable objects are discussed hereinafter.

1) Heavy objects: Objects heavier than $350 \mathrm{~g}$ are typically not graspable with the $\mathrm{iCub}$ hand, even if their size would allow. The force exerted by the fingers is not enough to hold them against gravity. From Fig. 3 (left to right, top to bottom): cereal box, wood block, hammer, bleach cleanser, Windex bottle, skillet, skillet lid, Master Chef can, potted meat can, sugar box, power drill, mustard bottle.

2) Big objects: Although the maximum distance that can be obtained between the tip of the thumb and the tip of the index or middle fingers is about $120 \mathrm{~mm}$, objects larger than $100 \mathrm{~mm}$ in all the dimensions are typically not graspable, even if light enough. From Fig. 3: soccer ball.

3) Thin objects: Since we are considering the grasping of objects from the table, a minimum thickness of the object is required (about $15 \mathrm{~mm}$, according to our experiments). Objects that are too thin cannot be grasped. From Fig. 3 (left to right, top to bottom): adjustable wrench, scissors, nails, washers, small marker, spoon, blank credit card, fork, knife.

4) Other non-graspable objects: The yellow softball (191 g, $96 \mathrm{~mm}$, on the rightmost side in Fig. 3) has a size and a weight that should allow grasping (even if close to the limits), but it is too hard and smooth, and therefore it slips easily. The padlock (304 g) is also too hard and smooth to be held steadily against gravity.

The red plate and bowl (in the middle of Fig. 3) do not exceed in size and weight, but have shapes that are difficult to grasp: because of the excessive diameter they can only be grasped from one of the edges, and after grasping the center of mass of the object is too far from the hand, causing the object to slip (also because of the very smooth texture).

5) Borderline objects: Despite the binary classification into graspable and non-graspable, some objects are more difficult than others to grasp, and some might be considered borderline (they might be graspable under some conditions). For example, some objects that were successfully grasped in a few occasions (but that were typically not held steadily in the hand) have not been included in the graspable set because of their excessive weight, that can easily damage the hand: 


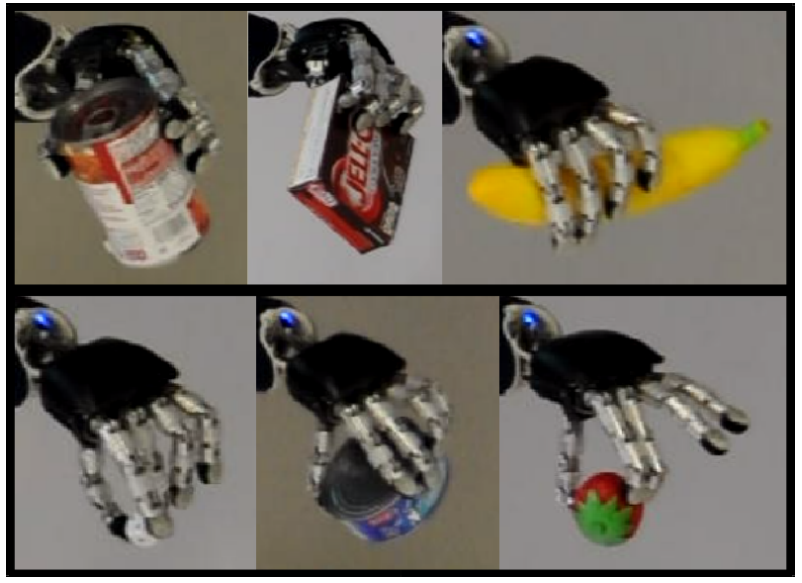

Fig. 5. Different grasp types used for different objects. From left to right, top to bottom: lateral cylindrical wrap (tomato soup can), lateral prismatic 5fingers grip (pudding box), top cylindrical wrap (banana), 2-fingers precision grip (dice), top prismatic 5-fingers grip (tuna can), 3-fingers precision grip (strawberry).

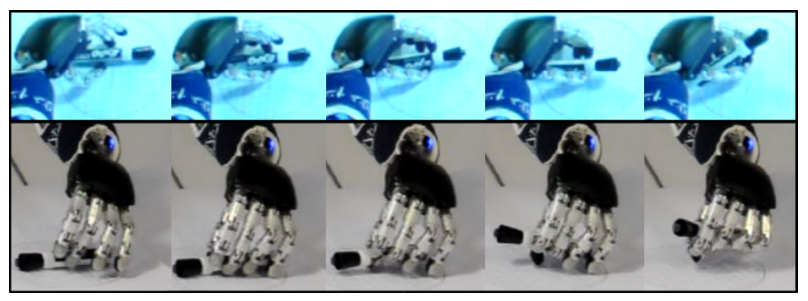

Fig. 6. Sequence of fingers movements to grasp the large marker. First the thumb and middle finger are used to raise the object from the table, then all the fingers are used to wrap it. Top row: images from one robot camera. Bottom row: external views.

the skillet lid, the crackers box, the sugar box and the potted meat can. The padlock cannot be grasped from its body, and therefore it was not included in the graspable set; however, the hook could be caged by the index and medium fingers and the thumb to safely lift the object.

Among the graspable objects, the pitcher base and pitcher lid can be grasped only using the appropriate handles (they cannot be grasped, for example, with a pinch grip on one of the edges). Thin elongated objects (e.g. the large marker, the banana, the spatula, the screwdrivers) can be grasped with the hand approaching from the top, with a complex motion that starts as a prismatic 5-fingers grip and then becomes a cylindrical wrap, after the object slips inside the fingers; the sequence of finger movements for the grasping of the large marker is shown in Fig. 6. Small thin objects that are very light and compact can be grasped with a 2-fingers precision grip, for example the plastic nut $(8 \times 15 \mathrm{~mm}, 1 \mathrm{~g})$, the smallest marble $(14 \mathrm{~mm}, 4 \mathrm{~g})$ and the dice $(16 \mathrm{~mm}, 5 \mathrm{~g})$; however, in these cases high precision is required in the hand positioning.

\section{B. Gripper Assessment Benchmarks}

Table I presents the scores measured on the Basic GAB: the total score is 173 (maximum score would be 228). The scores for the additional objects that are included in the Extended GAB are displayed in Table II. The total score considering also these objects is $337(173+164)$; maximum score would be 436 . These benchmarks provide a more indepth analysis on the grasping robustness with respect to representative objects of the set.

The iCub hand can reliably grasp round, cubic and cylindrical objects that fit the size and weight constraints. The bigger spheres (tennis ball and racquetball) can be grasped with a 3-fingers wrap (as in Fig. 1), the medium ones (golf ball and XL marble) with a 3-fingers precision grip (similar to the one used for the strawberry in Fig. 5) and the smaller ones (marbles of size L, M and S) with a 2-fingers precision grip (similar to the one used for the dice in Fig. 5). The 2 -fingers grip is indeed used for all very small objects. Big cubic objects can grasped with lateral prismatic grips (e.g. the pudding box in Fig. 5). Cylindrical objects can be grasped with a lateral cylindrical wrap (similar to the one used for the tomato soup can in Fig. 5) or with a 3-fingers grip, if they are light enough (e.g. cups). Objects with complex shapes can be more challenging, but they are typically grasped if not too big or heavy (e.g. the strawberry in Fig. 5). Elongated objects (marker and screwdriver) can be grasped using the strategy described in Fig. 6. While the rope can be consistently wrapped by the fingers (with a top wrap), the chain very often cannot, due to its larger size.

For thin and/or small objects, displacements on the $\mathrm{Z}$ axis cannot be accommodated; the hand positioning needs to be very precise on the $\mathrm{Z}$ axis. Instead, small displacements on the $\mathrm{X}$ and $\mathrm{Y}$ can be compensated also for very small objects, by using different fingers for the grips (e.g. the index finger instead of the middle finger). However, objects that are closer to the maximum graspable size (chips can, glass and large cup) may not allow for displacements on the $\mathrm{X}$ or the $\mathrm{Y}$ axis. Objects that have a preferential grasping orientation (e.g. marker, screwdriver, XL clamp) or that are too large on one dimension (e.g. pudding box, pear) might not be successfully grasped when rotated $90^{\circ}$.

\section{Grasp Flexibility Benchmark}

The results of the GFB experiment are reported in Table III. The scores on the $\mathrm{X}$ and $\mathrm{Y}$ rows are relative to object displacements from $-5 \mathrm{~cm}$ to $+5 \mathrm{~cm}$, while the ones on the $\mathrm{Z}$ row are for displacements from $-2 \mathrm{~cm}$ to $+2 \mathrm{~cm}$. The total score is 126 (the maximum score would be 200). We executed the benchmark on the foam brick object because it has the average size and weight that the iCub can easily grasp, and its cubic shape allows to precisely set the position and orientation at each grasp; moreover, its softness allows to test the dexterity of the iCub hand with no risks of breakages. 
TABLE I

SCORING OF THE ICUB HAND IN THE BASIC GRIPPER ASSESSMENT BENCHMARK (A.O. STANDS FOR ARTICULATED OBJECTS). TOTAL SCORE WAS 173 (MAXIMUM: 228).

\begin{tabular}{|c|c|c|c|c|c|}
\hline & Object & SP1 & SP2 & SP3 & SP4 \\
\hline \multirow{7}{*}{ 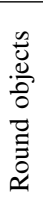 } & Tennis ball & 4 & 4 & 4 & 4 \\
\hline & Racquetball & 4 & 4 & 4 & 4 \\
\hline & Golf ball & 4 & 4 & 4 & 4 \\
\hline & Marble XL & 4 & 4 & 4 & 4 \\
\hline & Marble L & 4 & 4 & 4 & 0 \\
\hline & Marble M & 4 & 4 & 4 & 0 \\
\hline & Marble S & 4 & 4 & 4 & 0 \\
\hline \multirow{6}{*}{$\frac{0}{8}$} & Large marker & 2 & 2 & 2 & 0 \\
\hline & Flat screwdriver & 2 & 2 & 2 & 0 \\
\hline & Clamp XL & 2 & 2 & 2 & 2 \\
\hline & Clamp L & 4 & 4 & 4 & 4 \\
\hline & Clamp M & 4 & 4 & 4 & 0 \\
\hline & Clamp S & 4 & 4 & 4 & 0 \\
\hline & Rope & 10 & - & - & - \\
\hline 这 & Chain & 3 & - & - & - \\
\hline
\end{tabular}

TABLE II

SCORING OF THE ICUB HAND WITH THE ADDITIONAL OBJECTS INCLUDED IN THE EXTENDED GRIPPER ASSESSMENT BENCHMARK. TOTAL SCORE ON THESE OBJECTS WAS 164 . ADDED TO THE SCORE IN THE BASIC GAP, THE OVERALL SCORE OF THE ICUB HAND WAS 337 (MAXIMUM: 436).

\begin{tabular}{|c|c|c|c|c|c|}
\hline & Object & SP1 & SP2 & SP3 & SP4 \\
\hline \multirow{4}{*}{ U. } & Pudding box & 2 & 2 & 2 & 2 \\
\hline & Foam brick & 4 & 4 & 4 & 4 \\
\hline & Colored wood block & 4 & 4 & 4 & 4 \\
\hline & Dice & 4 & 4 & 4 & 0 \\
\hline \multirow{5}{*}{ 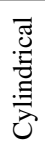 } & Chips can & 4 & 4 & 0 & 4 \\
\hline & Plastic wine glass & 4 & 4 & 0 & 4 \\
\hline & Large cup & 4 & 0 & 0 & 4 \\
\hline & Medium cup & 4 & 4 & 4 & 4 \\
\hline & Small cup & 4 & 4 & 4 & 4 \\
\hline \multirow{4}{*}{ ن } & Plastic pear & 2 & 2 & 2 & 2 \\
\hline & Plastic strawberry & 4 & 4 & 4 & 4 \\
\hline & Plastic bolt & 4 & 4 & 4 & 0 \\
\hline & Plastic nut & 4 & 4 & 4 & 0 \\
\hline
\end{tabular}

Some of the object poses used in the experiment are shown in Fig. 7.

The scores we obtained show that considerable displacements of the object from the nominal pose can be accounted for. In particular, we observed that displacements of $3 \mathrm{~cm}$ or less did not typically jeopardize the grasping performance, and that in a few cases successful (or partially successful) grasps were achieved even in presence of larger displacements. In real world tasks, these displacements can be either real displacements of the object (undesired and/or unexpected), errors in the object pose estimation (due to sensor noise and/or occlusions) or errors in the hand positioning (due to inaccurate calibration and/or noise in the actuation). Similar considerations hold for the changes in the object orientation. Depending on the actual pose of the object, different fingers and different grasp types were used, exploiting the flexibility offered by the 9 DOFs of the iCub hand (some of the grasps are shown in Fig. 8).
TABLE III

SCORING OF THE ICUB HAND IN THE GRIPPER FLEXIBILITY BENCHMARK PERFORMED ON THE FOAM BRICK OBJECT. TOTAL SCORE IS 126 (MAXIMUM: 200).

\begin{tabular}{|c|c|c|c|c|}
\hline & $0^{\circ}$ & $45^{\circ}$ & $90^{\circ}$ & $135^{\circ}$ \\
\hline SP1 & 2 & 2 & 2 & 2 \\
\hline \multirow{2}{*}{$\mathrm{X}$} & {$[2,2,2,2,2$,} & {$[0,2,2,2,2$,} & {$[0,1,2,2,2$,} & {$[0,1,2,2,2$,} \\
& $2,2,2,0,0]$ & $2,2,2,1,0]$ & $2,2,2,1,0]$ & $2,2,2,1,0]$ \\
\hline \multirow{2}{*}{$\mathrm{Y}$} & {$[0,1,2,2,2$,} & {$[0,1,2,2,2$,} & {$[0,0,0,1,2$,} & {$[0,1,2,2,2$,} \\
& $2,2,0,0,0]$ & $2,2,2,1,0]$ & $2,1,0,0,0]$ & $2,2,1,0,0]$ \\
\hline $\mathrm{Z}$ & {$[2,2,2,2]$} & {$[2,2,2,2]$} & {$[2,2,2,2]$} & {$[2,2,2,2]$} \\
\hline
\end{tabular}

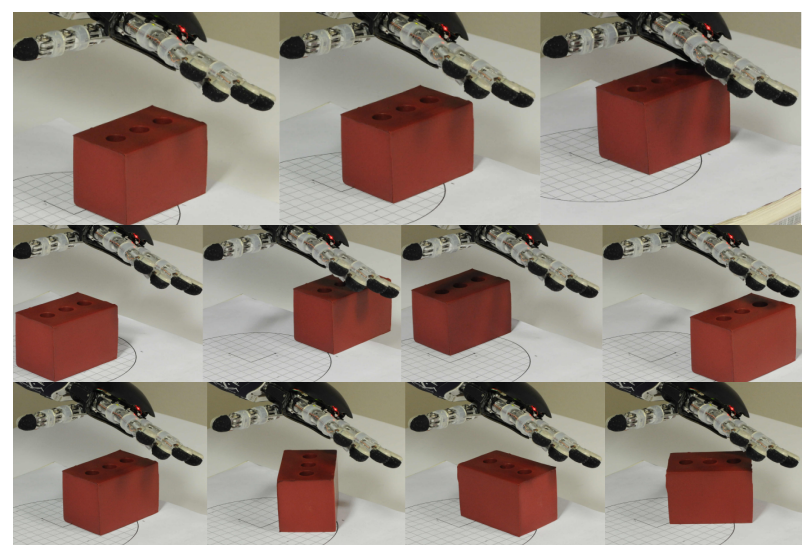

Fig. 7. Some of the object poses used in the GFB. From left to right. First row: displacements of $-2 \mathrm{~cm}, 0 \mathrm{~cm}, 2 \mathrm{~cm}$ on $\mathrm{Z}$ (the middle image corresponds to SP1). Second row: maximum displacements on $X$ and $Y( \pm 5 \mathrm{~cm})$. Third row: the four orientations used for each position: $0^{\circ}, 45^{\circ}, 90^{\circ}$ and $135^{\circ}$.

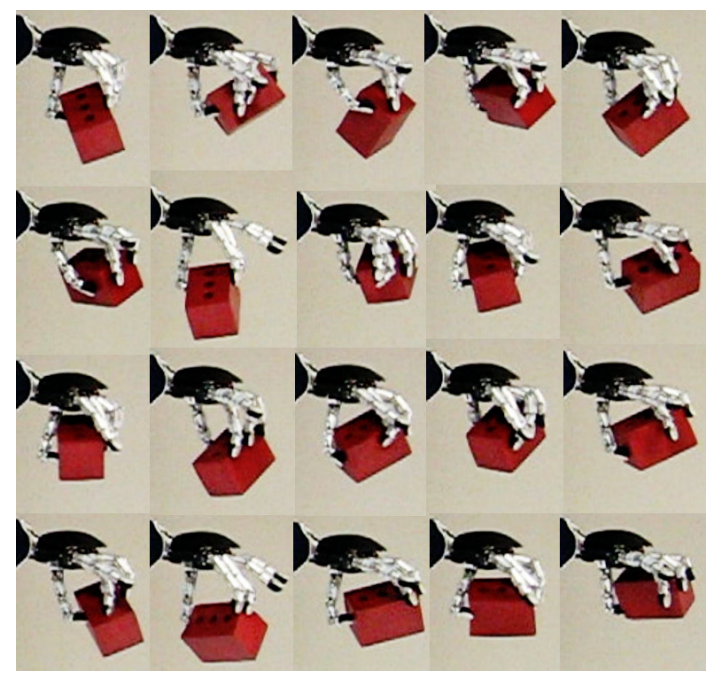

Fig. 8. Some exemplar grasps used in the GFB experiment.

This is a clear advantage with respect to simple grippers; however, the presence of many DOFs must be supported by a sophisticated controller to be a real advantage in practical situations. 
IEEE ROBOTICS AND AUTOMATION LETTERS. PREPRINT VERSION. ACCEPTED DECEMBER, 2015

\section{CONCLUSIONS AND FUTURE WORK}

In this paper we perform an evaluation of the grasping capabilities of the iCub hand using the YCB Object and Models Set. We experimentally identify a subset of the YCB Set comprising objects that are graspable with the iCub hand, and we perform three different benchmarks on some of those objects, showcasing the grasping capabilities of the robot. An expert human user controls the fingers motion using the CyberGlove-II dataglove; ideally, this provides the robot with the best possible controller (i.e. the human brain), and therefore it allows to generate a performance baseline that researchers can compare to. We offer a useful resource for the iCub community, and also suggest a general methodology for benchmarking in grasping and manipulation research. Based on our experiments we also discuss how the use of articulated hands with many DOFs may impact the robustness and flexibility of grasping, if a smart controller is available. In this work we focus on the mechanical possibilities of the iCub hand, exploiting the human brain as a controller and the human vision as a sensory feedback. We do not consider tactile perception, nor the human or the robot one. An interesting addition to this work would be to include tactile sensing, providing also tactile feedback to the human operator (for example, using a dataglove similar to the prototype presented in [25]). This would allow more complex benchmarking, possibly exploring also the implications of using either vision alone, touch alone, or the two modalities combined.

\section{REFERENCES}

[1] M. Helmert, "Complexity results for standard benchmark domains in planning," Artificial Intelligence, vol. 143, pp. 219-262, 2003.

[2] K. Gauman and B. Leibe, Visual Object Recognition. Morgan \& Claypool Publishers, 2010.

[3] P. Dollar, C. Wojek, B. Schiele, and P. Perona, "Pedestrian detection: An evaluation of the state of the art," Pattern Analysis and Machine Intelligence, IEEE Transactions on, vol. 34, no. 4, pp. 743-761, 2012.

[4] A. P. del Pobil, R. Madhavan, and E. Messina, "Benchmarks in robotics research," in IEEE IROS Workshop on Benchmarks in Robotics Research, 2006.

[5] B. Calli, A. Walsman, A. Singh, S. Srinivasa, P. Abbeel, and A. M. Dollar, "Benchmarking in manipulation research: The ycb object and model set and benchmarking protocols," arXiv preprint arXiv:1502.03143, 2015.

[6] B. Calli, A. Singh, A. Walsman, S. Srinivasa, P. Abbeel, and A. M. Dollar, "The ycb object and model set: Towards common benchmarks for manipulation research," in IEEE International Conference on Advanced Robotics (ICAR), 2015.

[7] G. Metta, L. Natale, F. Nori, G. Sandini, D. Vernon, L. Fadiga, C. von Hofsten, K. Rosander, M. Lopes, J. Santos-Victor, A. Bernardino, and L. Montesano, "The icub humanoid robot: An open-systems platform for research in cognitive development," Neural Networks, vol. 23, no. 89, pp. 1125-1134, 2010.
[8] Y. Su, Y. Wu, K. Lee, Z. Du, and Y. Demiris, "Robust grasping for an under-actuated anthropomorphic hand under object position uncertainty," in IEEE International Conference on Humanoid Robots (Humanoids), Nov 2012, pp. 719-725.

[9] B. Huang, S. El-Khoury, M. Li, J. Bryson, and A. Billard, "Learning a real time grasping strategy," in IEEE International Conference on Robotics and Automation (ICRA), May 2013, pp. 593-600.

[10] A. Bernardino, M. Henriques, N. Hendrich, and J. Zhang, "Precision grasp synergies for dexterous robotic hands," in IEEE International Conference on Robotics and Biomimetics (ROBIO), Dec 2013, pp. 6267.

[11] J. Leitner, M. Frank, A. Forster, and J. Schmidhuber, "Reactive reaching and grasping on a humanoid: Towards closing the action-perception loop on the icub," in International Conference on Informatics in Control, Automation and Robotics (ICINCO), vol. 01, Sept 2014, pp. 102-109.

[12] G. Cotugno, V. Mohan, K. Althoefer, and T. Nanayakkara, "Simplifying grasping complexity through generalization of kinaesthetically learned synergies," in IEEE International Conference on Robotics and Automation (ICRA), May 2014, pp. 5345-5351.

[13] J.-P. Saut, S. Ivaldi, A. Sahbani, and P. Bidaud, "Grasping objects localized from uncertain point cloud data," Robotics and Autonomous Systems, vol. 62, no. 12, pp. 1742-1754, 2014.

[14] I. Gori, U. Pattacini, V. Tikhanoff, and G. Metta, "Ranking the good points: A comprehensive method for humanoid robots to grasp unknown objects," in IEEE International Conference on Advanced Robotics (ICAR), November 2013, pp. 1-7.

[15] _ - "Three-finger precision grasp on incomplete $3 \mathrm{~d}$ point clouds," in IEEE International Conference on Robotics and Automation (ICRA), May 2014, pp. 5366-5373.

[16] P. Fitzpatrick, G. Metta, and L. Natale, "Towards long-lived robot genes," Robotics and Autonomous Systems, vol. 56, no. 1, pp. 29-45, 2008.

[17] "Robotology," https://github.com/robotology, accessed: 19-08-2015.

[18] A. Parmiggiani, M. Maggiali, L. Natale, F. Nori, A. Schmitz, N. Tsagarakis, J. Santos-Victor, F. Becchi, G. Sandini, and G. Metta, "The design of the icub humanoid robot," International Journal of Humanoid Robotics, vol. 09, no. 04, p. 1250027, 2012.

[19] A. Schmitz, U. Pattacini, F. Nori, L. Natale, G. Metta, and G. Sandini, "Design, realization and sensorization of the dexterous icub hand," in IEEE International Conference on Humanoid Robots (Humanoids), Dec 2010, pp. 186-191.

[20] L. Dipietro, A. Sabatini, and P. Dario, "A survey of glove-based systems and their applications," IEEE Transactions on Systems, Man, and Cybernetics, Part C: Applications and Reviews, vol. 38, no. 4, pp. 461-482, 2008.

[21] M. Diftler, C. Culbert, R. Ambrose, J. Platt, R., and W. Bluethmann, "Evolution of the nasa/darpa robonaut control system," in IEEE International Conference on Robotics and Automation, vol. 2, 2003, pp. 2543-2548.

[22] F. Kobayashi, K. Kitabayashi, H. Nakamoto, F. Kojima, W. Fukui, N. Imamura, and T. Maeda, "Multiple joints reference for robot finger control in robot hand teleoperation," in IEEE/SICE International Symposium on System Integration, 2012, pp. 577-582.

[23] G. Saggio and M. Bizzarri, "Feasibility of teleoperations with multifingered robotic hand for safe extravehicular manipulations," Aerospace Science and Technology, vol. 39, pp. 666 - 674, 2014.

[24] "Immersion CyberGlove-II," http://www.immersion.fr/en/cybergloveii/, accessed: 19-08-2015.

[25] J. Lobo and P. Trindade, "Inertouchhand system - ith - demonstration of a glove device with distributed inertial sensors and vibro-tactile feedback," International Journal of Online Engineering, vol. 9, no. S8, pp. 56-58, 2013. 